\title{
Efficacy of usual antidepressant dosing regimens of fluoxetine in panic disorder
}

\author{
Randomised, placebo-controlled trial \\ D. MICHELSON, C. ALLGULANDER, K. DANTENDORFER, A. KNEZEVIC, \\ D. MAIERHOFER, V. MICEV, V. R. PAUNOVIC, I. TIMOTIJEVIC, N. SARKAR, \\ L. SKOGLUND and S. C. PEMBERTON
}

\author{
Background Although serotonin \\ reuptake inhibitors are effective in panic \\ disorder, questions concerning whether \\ doses associated with antidepressant \\ efficacy are also effective for panic \\ disorder remain.
}

Aims To assess the efficacy of the usual antidepressant dose of fluoxetine in treating full panic attacks.

\begin{abstract}
Method Patients with panic disorder were randomised to placebo or to fluoxetine initiated at $10 \mathrm{mg}$ daily for I week and then increased to $20 \mathrm{mg}$ daily. The trial lasted 12 weeks, but after 6 weeks patients who had failed to achieve a satisfactory response were eligible for dose escalation to a maximum of $60 \mathrm{mg}$ of fluoxetine daily.
\end{abstract}

\section{Results Fluoxetine was associated with a statistically significantly greater proportion of panic-free patients compared with placebo after 6 weeks and at end-point.}

\section{Conclusions Fluoxetine at a dose of $20 \mathrm{mg}$ daily is safe and efficacious in reducing symptoms of panic disorder. Patients who fail to obtain a satisfactory response at $20 \mathrm{mg}$ daily may benefit from further dose increases.}

\footnotetext{
Declaration of interest This study was funded by Eli Lilly and Company. Drs Michelson and Sarkar and Mr Pemberton are employees of Eli Lilly and Company. Drs Allgulander, Dantendorfer, Knezevic, Maierhofer, Micev, Paunovic, Timotijevic and Skoglund are paid consultants to Eli Lilly and Company.
}

Few data are available concerning doseresponse and minimal effective dose of selective serotonin reuptake inhibitors for treatment of panic disorder. Data are conflicting as to whether doses associated with antidepressant efficacy are also effective in treatment panic disorder (Wade et al, 1997; Ballenger et al, 1998; Londborg et al, 1998). In one acute, randomised trial of fluoxetine in panic disorder, $10 \mathrm{mg}$ daily showed superiority to placebo for some symptoms, whereas $20 \mathrm{mg}$ daily showed superiority across a broader range of symptoms (Michelson et al, 1998). Superiority to placebo in reduction of full panic attack frequency was not demonstrated at either dose. This could have been related to methodological issues associated with measuring panic frequency. Alternatively, higher doses may be necessary to achieve a specific effect on panic frequency. We report here a study of fluoxetine treatment for panic disorder incorporating an initial dose of $20 \mathrm{mg}$ daily, followed by titration of dose upwards for patients who failed to achieve a satisfactory response.

\section{METHOD}

\section{Patient population}

Participants in the trial were men and women aged at least 18 years who met DSM-IV (American Psychiatric Association, 1994) criteria for panic disorder as assessed by the structured clinical interview for DSM-IV (SCID; First et al, 1995). Patients had to have had a minimum of four full panic attacks in the month preceding entry into the study and two full panic attacks during the 2-week baseline evaluation period. Additionally, patients had to have at least moderate symptom severity, as indicated by a Panic Disorder Severity Scale (PDSS; Shear et al, 1997) score of 12 or greater and a Clinical Global Impression of Severity (CGI-Severity; Guy, 1976) rating of 4 or greater. Patients who met DSM-IV criteria for any other concurrent anxiety disorder or for major depression or had a 17-item Hamilton Rating Scale for Depression (HRSD; Hamilton, 1960) score $\geqslant 22$ were ineligible for the study, as were patients with current or recent alcohol or substance misuse or dependence. Patients who previously had a trial of at least 6 weeks with one of the following agents at or above the noted doses without significant benefit also were excluded from the trial: fluoxetine, $40 \mathrm{mg}$; sertraline, $100 \mathrm{mg}$; paroxetine, $40 \mathrm{mg}$; clomipramine, $150 \mathrm{mg}$; citalopram, $40 \mathrm{mg}$. The study was conducted on out-patients at nine sites in Europe. Subjects were recruited from patients who presented for evaluation at psychiatric clinics, by referral and by advertisement. The ethical review board for each site reviewed the study and written informed consent was obtained from all participants.

\section{Study design}

Following a 2-week medication washout period, the study incorporated an initial single-blind (patients) 2-week baseline evaluation period followed by randomisation under double-blind conditions to either fluoxetine or placebo for 12 weeks. Dosing was initiated at $10 \mathrm{mg}$ daily for 1 week and then increased to $20 \mathrm{mg}$ daily. After 6 weeks, patients who had not achieved a CGI-Severity score of 2 or less (minimal symptoms) were required to have a dosage increase to $40 \mathrm{mg}$. If CGI-Severity at subsequent visits remained above 2 (indicating some residual symptoms), an additional increase to $60 \mathrm{mg}$ was required.

Patients were seen at 2 -week intervals throughout the study. Panic frequency was assessed using an electronic diary that was completed by patients after each panic attack and included information on duration, severity and whether the attack was 'spontaneous' or triggered by an external stimulus, as well as which symptoms were experienced during the attack. Other assessments included the PDSS, the 17-item HRSD, the Hamilton Rating Scale for Anxiety (HRSA; Riskind et al, 1987), the State Anxiety Inventory (SAI; Spielberger, 1983) and CGI-Severity. The Sheehan Disability Scales (Sheehan, 1983) were used to assess patients' functional impairment.

\section{Statistical methods}

All the analyses were based upon the intention-to-treat principle. The sample size of 180 patients was calculated to provide a 
$90 \%$ likelihood of detecting a $20 \%$ difference between treatment groups in panic-free status at end-point (the protocol-specified primary outcome measure). Efficacy evaluations were made at 6 weeks and at end-point for measures of panic frequency and the PDSS. Other measures were assessed at end-point only. SAS software, version 6.09 (SAS Institute, 1991) was used for all analyses. Logistic regression methods were used to compare fluoxetine and placebo groups after 6 weeks and during the final visit interval with respect to the odds of being panic-free and of having a $50 \%$ reduction in panic frequency, and during the final visit interval with respect to the odds of having a final CGI-Severity score of 1 or 2 . The logistic regression model included treatment, site and treatment-site interaction (with provision to delete treatment-site from the model if the term was non-significant). Likelihood ratio tests were used to test the treatment effects. The GENMOD procedure of the SAS system was used for logistic regression analyses.

Continuous data (PDSS, HRSD, HRSA, Sheehan Disability Scale, SAI and CGISeverity) were analysed using an analysis of variance to test the null hypothesis of no difference in mean change from baseline to end-point between groups. Prior to analysis, panic frequency was rank-transformed because of non-normality of the data. Adverse events were compared between groups using Fisher's exact test.

\section{RESULTS}

\section{Patient characteristics and baseline severity}

Among 207 patients evaluated for the study, 180 met eligibility criteria, were randomised to either fluoxetine $(n=90)$ or placebo $(n=$ 90), had at least one visit following randomisation and were included in outcome analyses. Demographic data, baseline panic attack frequency, proportion of patients with agoraphobia and symptom severity were similar for both groups (Table 1).

\section{Efficacy}

Outcomes are summarised in Table 2. For the protocol-specified primary outcome measure (panic-free status at end-point), analysis did not demonstrate a treatmentsite interaction for the logistic regression comparison of likelihood ratios, and this term was dropped from the model. Patients treated with fluoxetine achieved significantly greater improvements than the placebo group at 6 weeks and at end-point in panic-free status, $50 \%$ or more reduction in panic attacks and proportion of patients with CGI-Severity scores or 1 or 2 (no or minimal symptoms). The estimated odds of achieving panic-free status at 6 weeks and end-point were $2.38(95 \% \mathrm{CI}=$ 1.11-5.11) and 2.29 (95\% CI=1.14-4.59) times higher, respectively, for fluoxetinetreated patients compared with patients receiving placebo. At 6 weeks, $29 \%$ of fluoxetine patients and $16 \%$ of patients in the placebo group were panic free. Fortytwo per cent of fluoxetine-treated patients and $28 \%$ of patients in the placebo group were panic free at end-point. The estimated odds of achieving at least a $50 \%$ reduction from baseline in the number of full panic attacks at 6 weeks and at end-point were $1.94(95 \% \mathrm{CI}=1.04-3.62)$ and $3.23(95 \%$ $\mathrm{CI}=1.57-6.63)$ times higher, respectively, for fluoxetine-treated patients compared with patients in the placebo group. At 6 weeks, $61 \%$ of fluoxetine patients and $47 \%$ of placebo patients reported a $50 \%$ or greater reduction in the number of panic attacks. The percentages of patients who demonstrated at least a $50 \%$ reduction at end-point were $82 \%$ and $61 \%$ in the fluoxetine and placebo groups, respectively. Mean reduction in panic attack frequency as measured by diary was not statistically significantly different between the groups. However, mean reduction in panic attack frequency as assessed by item 1 of the PDSS was significantly greater among fluoxetine patients at both 6 weeks and end-point.

Table I Baseline characteristics and illness severity

\begin{tabular}{|c|c|c|}
\hline Demographic data & Fluoxetine $(n=90)$ & Placebo $(n=90)$ \\
\hline$\%$ Male/female & $48 / 52$ & $41 / 59$ \\
\hline Age (years) $)^{\prime}$ & $36.5(10.3)$ & $34.8(9.8)$ \\
\hline Full panic attacks per week' & $3.9(3.5)$ & $3.8(3.0)$ \\
\hline Panic Disorder Severity Scale ${ }^{\prime}$ & $18.4(3.4)$ & I8.I (3.3) \\
\hline Panic Disorder Severity Scale item I 1,2 & $2.9(0.7)$ & $2.8(0.6)$ \\
\hline Clinical Global Impression of Severity' & $5.1(0.8)$ & $5.1(0.8)$ \\
\hline Hamilton Rating Scale for Anxiety' & $23.1(7.3)$ & $23.6(6.7)$ \\
\hline State Anxiety Inventory' & $53.7(13.3)$ & $52.3(12.3)$ \\
\hline Hamilton Rating Scale for Depression' & $10.9(4.0)$ & II.6 (4.3) \\
\hline Sheehan Work Impairment' & $6.6(2.5)$ & $6.5(2.6)$ \\
\hline Sheehan Social Impairment ${ }^{1}$ & $6.6(2.3)$ & $6.9(2.0)$ \\
\hline Sheehan Family' & $5.3(2.3)$ & $5.6(2.3)$ \\
\hline
\end{tabular}

I. Values are presented as mean (s.d.).

2. Item $I$ is panic attack frequency, including limited symptom episodes, rated from 0 (no panic or limited symptom episodes) to 4 (extreme - full panic attacks occur more than once a day, more days than not).
Reduction in global panic disorder symptom severity as assessed by the overall PDSS score was statistically significantly greater for fluoxetine-treated patients than for those treated with placebo. More general measures of anxiety, including the HRSA and SAI, also demonstrated greater symptom reduction by fluoxetine compared with placebo, as did measures of functional impairment, including the Sheehan work and social impairment measures and the social impairment item of the PDSS.

The final median dose of the fluoxetine treatment group was $20 \mathrm{mg}$ daily and the final mean dose was $29.8 \mathrm{mg}$ daily. Among fluoxetine patients at end-point, 61 subjects $(67.7 \%)$ were taking $20 \mathrm{mg}$ daily, 14 $(15.5 \%)$ were taking $40 \mathrm{mg}$ daily and 15 (16.7\%) were taking $60 \mathrm{mg}$ daily.

\section{Safety}

Among randomised patients, the number of patients reaching the final visit after 12 weeks of fluoxetine or placebo therapy was similar for both groups (fluoxetine: $n=75 \quad(83.3 \%)$; placebo: $n=80 \quad(88.8 \%)$; NS). The total number of discontinuations due to adverse events was similar for both groups (fluoxetine: $n=5$ (5.5\%); placebo: $n=3(3.3 \%) ; P=0.72$, Fisher's exact test). Other reasons for discontinuation included lack of efficacy (fluoxetine: $n=5(5.5 \%)$; placebo: $n=3$ (3.3\%); $P=0.72$, Fisher's exact test), patients lost to follow-up (fluoxetine: $n=3(2.2 \%)$; placebo: $n=3(2.2 \%)$; $P=0.99$, Fisher's exact test), patient decision (fluoxetine: $n=2$ (2.2\%); placebo: $n=0$; $P=0.50$, Fisher's exact test) and protocol 


\begin{tabular}{|c|c|c|c|c|c|c|c|c|}
\hline & \multicolumn{4}{|c|}{ Six-week outcomes } & \multicolumn{4}{|c|}{ End-point outcomes } \\
\hline & Fluoxetine & Placebo & $F$ & $P$ & Fluoxetine & Placebo & $\mathrm{F}$ & $P$ \\
\hline Proportion panic free & $29 \%$ & $16 \%$ & & $0.024^{\prime}$ & $42 \%$ & $28 \%$ & & $0.02^{\prime}$ \\
\hline Proportion with $50 \%$ reduction & $61 \%$ & $47 \%$ & & $0.036^{2}$ & $82 \%$ & $61 \%$ & & $0.001^{2}$ \\
\hline Panic frequency (reduction) ${ }^{3}$ & $-2.3(3.0)$ & $-I .5(3.1)$ & 2.95 & $0.088^{4}$ & $-2.9(3.2)$ & $-2.2(3.2)$ & 1.04 & $0.31^{4}$ \\
\hline Panic reduction (PDSS item I) ${ }^{3}$ & $-1.0(1.0)$ & $-0.7(0.9)$ & 5.03 & $0.026^{4}$ & $-1.7(I . I)$ & $-1.1(I . I)$ & 7.20 & $0.008^{4}$ \\
\hline PDSS overall score (reduction) ${ }^{3}$ & $-6.8(5.3)$ & $-4.9(4.5)$ & 4.52 & $0.035^{4}$ & $-11.5(6.5)$ & $-7.6(6.3)$ & 6.93 & $0.009^{4}$ \\
\hline \multicolumn{9}{|l|}{ Proportion with CGI-Severity score of I or 2} \\
\hline at end-point & & & & & $65 \%$ & $37 \%$ & & $<0.001^{5}$ \\
\hline Hamilton Rating Scale for Anxiety (reduction) ${ }^{3}$ & & & & & $-14.9(9.1)$ & $-10.0(9.9)$ & 2.23 & $0.043^{4}$ \\
\hline State Anxiety Inventory (reduction) ${ }^{3}$ & & & & & $-15.3(13.6)$ & $-7.5(14.6)$ & 8.19 & $0.005^{4}$ \\
\hline Hamilton Rating Scale for Depression (reduction) ${ }^{3}$ & & & & & $-6.5(4.7)$ & $-4.2(5.8)$ & 2.23 & $0.14^{4}$ \\
\hline Sheehan Work (reduction) ${ }^{3}$ & & & & & $-4.1 \quad(3.0)$ & $-2.5(3.4)$ & 8.39 & $0.004^{4}$ \\
\hline Sheehan Family (reduction) ${ }^{3}$ & & & & & $-3.6(2.7)$ & $-2.6(3.4)$ & 2.36 & $0.13^{4}$ \\
\hline Sheehan Social Impairment (reduction) ${ }^{3}$ & & & & & $-4.2(2.9)$ & $-2.8(3.5)$ & 10.33 & $0.002^{4}$ \\
\hline
\end{tabular}

PDSS, Panic Disorder Severity Scale; CGI-Severity, Clinical Global Impression of Severity.

I. Logistic regression: 6-week comparison odds ratio=2.38,95\% Cl I.II-5.II; end-point comparison odds ratio $=2.29,95 \% \mathrm{Cl}$ I.14-4.59.

2. Logistic regression: 6 -week comparison odds ratio=1.94, $95 \% \mathrm{Cl}$ I.04-3.62; end-point comparison odds ratio $=3.23,95 \% \mathrm{Cl} 1.57-6.63$.

3. Values are mean change from baseline (s.d.)

4. Two-way analysis of variance, dependent variable change from baseline to end-point.

5. Logistic regression: end-point comparison odds ratio=3.7I, 95\% Cl I.93-7.13.

requirement (fluoxetine: $n=1 \quad(1.1 \%)$; placebo: $n=2 \quad(2.2 \%) ; P=0.99$, Fisher's exact test). The total number of adverse events reported was similar for both groups (fluoxetine: $n=25$ (27.8\%); placebo: $n=19$ (21.1\%); $P=0.37$, Fisher's exact test). No single adverse event occurred in more than $5 \%$ of patients in either group, and no single event was statistically significantly more common among patients in either group (summarised in Table 3 ).

\section{DISCUSSION}

Selective serotonin reuptake inhibitors have become first-line therapies for the treatment of panic disorder and specific effects have been demonstrated on panic attack frequency in a number of studies (Black et al, 1993; Hoehn-Saric et al, 1993; Oehrberg et al, 1995). Data are conflicting with respect to whether the usual doses associated with antidepressant efficacy are effective in treating panic disorder or whether higher doses are required. Paroxetine, for example, was shown to be efficacious at $40 \mathrm{mg}$ but not at $20 \mathrm{mg}$ daily (Ballenger $e t$ al, 1998). One study of three fixed doses of sertraline $(50,100$ and $200 \mathrm{mg}$ daily) showed efficacy only for the pooled group, and not for any single dose compared with placebo (Londborg et al, 1998), whereas a

Table 3 Overall new and worsened adverse events during treatment and five most common individual adverse events reported by fluoxetine-treated patients

\begin{tabular}{lccc}
\hline Event & $\begin{array}{c}\text { Fluoxetine }(n=90) \\
(n, \%)\end{array}$ & $\begin{array}{c}\text { Placebo }(n=90) \\
(n, \%)\end{array}$ & $P$ \\
\hline Patients reporting: & & & \\
One or more adverse events & $25(27.8 \%)$ & $19(21.1 \%)$ & 0.39 \\
Nausea & $4(4.4 \%)$ & $3(3.3 \%)$ & 0.99 \\
Tremor & $4(4.4 \%)$ & 0 & 0.12 \\
Headache & $3(3.3 \%)$ & $4(4.4 \%)$ & 0.99 \\
Vomiting & $3(3.3 \%)$ & 0 & 0.25 \\
Insomnia & $3(3.3 \%)$ & $2(2.2 \%)$ & 0.99 \\
\hline
\end{tabular}

I. Fisher's exact test.

combined analysis of two studies suggested that doses of sertraline from 50 to $150 \mathrm{mg}$ daily were all associated with a reduction in panic frequency and did not show evidence of a dose response (Sheikh et al, 2000). Citalopram has been reported to be efficacious at $20-30 \mathrm{mg}$ or $40-60 \mathrm{mg}$ daily but not at $10-15 \mathrm{mg}$ daily (Wade et al, 1997), but these results are difficult to interpret because the study used a single item from a scale designed for other purposes to measure panic frequency, and had no specific panic assessments.

We previously reported evidence of efficacy for fluoxetine at both 10 and $20 \mathrm{mg}$ daily compared with placebo (Michelson et al, 1998), with evidence of a somewhat more robust pattern of response at $20 \mathrm{mg}$. In that study, however, neither dose was associated with an effect on full panic attack frequency. This finding could have been related to methodological issues associated with measuring panic attack frequency (Michelson et al, 1998) or to a need for higher doses to achieve a specific effect on panic attack frequency.

\section{Efficacy of fluoxetine}

The results of this study demonstrate a statistically significant difference in treatment effects on multiple measures of panic attack frequency after 6 weeks (when all 
patients were being treated with fluoxetine $20 \mathrm{mg}$ daily) and after 12 weeks (when daily doses ranged from $20 \mathrm{mg}$ to $60 \mathrm{mg}$ ). The initial 6-week period provided an opportunity to assess whether the usual antidepressant dose of fluoxetine is effective in treating full panic attacks as well as other symptoms of panic disorder. The study also provided an opportunity to determine whether the number of patients who require higher doses for optimal response is similar or dissimilar to that observed among patients with depression.

Among patients who met DSM-IV criteria for panic disorder, fluoxetine was associated with statistically significant improvement in panic attack symptoms and a reduction in broader symptom measures both after 6 weeks of treatment with $20 \mathrm{mg}$ daily and at end-point at doses up to $60 \mathrm{mg}$ daily. These data provide further evidence that $20 \mathrm{mg}$ is an efficacious dose in this disorder and suggest that the failure to demonstrate effects on panic attack frequency in the previous study was related to methodological issues rather than a need for higher doses of fluoxetine.

This study did not include an active comparator. The difference in numbers of panic-free patients observed in this study is comparable to that reported in some studies of other selective serotonin reuptake inhibitors (Oehrberg et al, 1995; Londborg, 1998; Pohl et al, 1998). Other studies, however, have failed to show a difference in panic-free status at end-point (Pollack et al, 1998) or have shown greater treatment differences for this variable between active drug and placebo (Ballenger et al, 1998). In this regard, we note that response rates are determined by many study-specific factors as well as by a drug's intrinsic effects, and that comparisons across different studies conducted under unique protocols and circumstances are difficult to interpret meaningfully. As a result, we cannot determine definitively from this study the relative effects of fluoxetine compared with other agents that have been reported to be efficacious.

A further question concerns the degree to which the observed statistically significant effects also represent clinically important change. Although the absolute response rate was high $(82 \%$ of fluoxetine-treated patients had at least a $50 \%$ decrease in panic frequency at end-point), placebo response (i.e. improvement related to non-specific interventions) also was high $(61 \%$ of patients assigned to placebo also had a
$50 \%$ reduction in panic frequency). This is not unusual, because panic symptoms often are quite placebo-responsive and the magnitudes of observed treatment differences between active drugs and placebo can be limited. However, the clinical trial itself can affect non-specific responses through such variables as subject selection, visit frequency, intensity of evaluations and patient expectations of a novel or experimental treatment, making extrapolation to other settings difficult. Further, the clinical relevance of an effect depends not only on its magnitude but also on a number of other variables, including the discomfort and impairment associated with the disorder, the natural history of the disorder and any burdens associated with therapy. The results of measures such as the Sheehan Disability Scale that favour fluoxetine suggest that the greater symptomatic improvement observed among fluoxetinetreated patients was associated with improved functional outcomes compared with placebo. However, the study was designed primarily to assess efficacy (i.e. to determine whether or not fluoxetine has specific effects in this disorder compared with placebo) and definitive assessment of the clinical relevance of these effects will require further studies with designs specific to this issue.

Although dose-escalation designs can confound dose and time effects, it seems likely that responses observed after only 6 weeks among patients who had not responded initially are attributable more to increased dose than to time, because fluoxetine would have been at steady state and expected to have induced relevant physiological changes in the brain homoeostasis well within that period. We therefore hypothesise that a subgroup of patients required doses above $20 \mathrm{mg}$ to attain optimal therapeutic responses. Overall, the final mean dose was $29.6 \mathrm{mg}$, a figure comparable to values found in many trials of similar design using fluoxetine in patients with depressive disorders (Beasley et al, 1991; Casacchia et al, 1993).

\section{Methodological issues}

Several methodological issues arose in the course of this study and are also of interest. In designing this study, we chose to use an electronic diary. Analyses were conducted directly on patient-entered data, unlike many previous studies in which results were reviewed, interpreted and subject to change based on the investigator's judgement. Because no control group with investigatorreviewed data was included, it is not possible to determine definitively which methodology provides more accurate data. We do note, however, that the results suggest that using patients' reports without investigator mediation provides sufficiently reliable data to demonstrate drug effects, because the diary reports are consistent with the investigator-administered assessments. In this context, item 1 of the PDSS, which is an investigator assessment of panic frequency based on a review of symptoms with the patient, demonstrated a similar outcome to the diary, although the PDSS results on that item were somewhat more robust in separating fluoxetine and placebo than the diary results (a finding potentially related to measurement issues associated with transforming panic attack frequency into a limited number of discrete categories).

We and others (Michelson et al, 1998) have suggested that, for multiple reasons, change in panic attack frequency is an unsatifactory method for assessing change in overall illness severity, and that measures that include panic attack frequency as well as a broader range of symptomatology appear to be more meaningful markers of change. In this study, two measures of effects on panic attacks (proportion of panic-free patients and $50 \%$ reduction in panic attack frequency) did show differences between fluoxetine and placebo. The treatment differences in mean change in panic attack frequency, although directionally consistent with the other measures, did not reach statistical significance. In this regard, the PDSS overall score has been suggested as a preferable alternative (Shear et al, 1997), because it reflects a multidimensional approach to assessment of panic disorder and has been validated psychometrically. The overall results of the PDSS and other measures (e.g. HRSA, Sheehan Disability Scale) provide evidence of fluoxetine-specific response across symptom domains other than panic associated with fluoxetine treatment, including improvement in illness-associated functional impairment.

\section{Tolerability and safety}

Fluoxetine was well tolerated in this study. Overall reports of adverse events were similar among both placebo- and fluoxetine-treated patients, and no individual adverse event was statistically 
significantly more frequent in the fluoxetine group. Discontinuations due to adverse events among both groups were also similar and low. Some authors have suggested that patients suffering from panic disorder could be particularly sensitive to initiating fluoxetine treatment and have advocated very low initial doses and gradual increases (Giesecke, 1990; Schneier et al, 1990). The results of this and previous work, however, provide evidence that the tolerability of fluoxetine among patients with panic disorder is similar to that among depressed patients.

\section{REFERENCES}

American Psychiatric Association (1994) Diagnostic and Statistical Manual of Mental Disorders (4th edn) (DSM-IV). Washington, DC: APA.

Ballenger, J. C., Wheadon, D. E., Steiner, M., et al (1998) Double-blind, fixed-dose, placebo-controlled study of paroxetine in the treatment of panic disorder. American Journal of Psychiatry, 155, 36-42.

Beasley, C. M., Jr., Dornseif, B. E., Pultz, J. A., et al (1991) Fluoxetine versus trazodone: efficacy and activating-sedating effects. Journal of Clinical Psychiatry, 52, 294-299.

Black, D. W., Wesner, R., Bowers, W., et al (1993) A comparison of fluoxamine, cognitive therapy, and placebo in the treatment of panic disorder. Archives of General Psychiatry, 50, 44-50.

Casacchia, A. E., Cassano, G. B., Faravelli, C., et al (1993) Double-blind study of the efficacy and safety of sertraline versus fluoxetine in major depression. International Clinical Psychopharmacology, 8, 197-202.

\section{First, M. B., Spitzer, R. I., Williams, J. B.W., et al} (1995) Structured Clinical Interview for DSM-IV (SCID-I) User's Guide and Interview, Research Version. New York: Biometrics Research Department, New York Psychiatric Institute.

Giesecke, M. E. (1990) Overcoming hypersensitivity to fluoxetine in a patient with panic disorder. American Journal of Psychiatry, 147, 532-533.

Guy, W. (1976) ECDEU Assessment Manual for Psychopharmacology. Revised DHEW Pub. (ADM). Rockville, MD: National Institute for Mental Health.

Hamilton, M. (1960) A rating scale for depression. Journal of Neurology, Neurosurgery and Psychiatry, 23, 56-62.

Hoehn-Saric, R., McLeod, D. R. \& Hipsley, P. A. (1993) Effect of fluvoxamine on panic disorder. Journal of Clinical Psychopharmacology, 13, 321-326.

Londborg, P. D., Wolkow, R., Smith, W. T., et al (1998) Sertraline in the treatment of panic disorder. A multisite, double-blind, placebo-controlled, fixed-dose investigation. British Journal of Psychiatry, 173, 54-60.

\section{Michelson, D., Lydiard, R. B., Pollack, M. H., et al} (1998) Outcome assessment and clinical improvement in panic disorder: evidence from a randomized controlled trial of fluoxetine and placebo. The Fluoxetine Panic

\section{CLINICAL IMPLICATIONS}

- Compared with placebo, $20 \mathrm{mg}$ of fluoxetine daily is associated with statistically significantly greater reduction in multiple measures of panic attack frequency, as well as with reduction of other symptoms of panic disorder. Patients who fail to obtain a satisfactory response to $20 \mathrm{mg}$ of fluoxetine daily may benefit from further dose increases up to $60 \mathrm{mg}$ daily.

- Fluoxetine is effective in the treatment of panic disorder at a mean dose similar to that typically efficacious for treatment of depression.

Fluoxetine is well tolerated in this disorder.

\section{LIMITATIONS}

- The relative efficacy of $20 \mathrm{mg}$ of fluoxetine daily compared with higher doses was not assessed, and no active comparator was included.

Efficacy during longer-term treatment was not studied.

- Research conditions may not reflect naturalistic practice, and the finding of superiority relative to placebo in the reduction of panic disorder symptoms is a demonstration of a drug-specific effect and not necessarily an assessment of clinical significance.

DAVID MICHELSON, MD, CHRISTER ALLGULANDER, MD, KARL DANTENDORFER, MD, ALEKSANDAR KNEZEVIC, MD, DAGMAR MAIERHOFER, MD, VITOMIR MICEV, MD, VLADIMIR R. PAUNOVIC, MD, IVANA TIMOTIJEVIC, MD, NEENA SARKAR, PhD, LENNART SKOGLUND, MD, S. CRAIG PEMBERTON, Lilly Research Laboratories, Eli Lilly and Company, Lilly Corporate Center, Indianapolis, Indiana, USA

Correspondence: Dr David Michelson, Lilly Research Laboratories, Eli Lilly and Company, Lilly Corporate Center, Drop Code 6026, Indianapolis, IN 46285, USA. Tel: (317) 2776443; fax: (317) 2773262

(First received I5 August 2000, final revision 29 May 200I, accepted I4 June 200I)

Disorder Study Group. American Journal of Psychiatry, 155, 1570-1577

Oehrberg, S., Christiansen, P. E., Behnke, K., et a (1995) Paroxetine in the treatment of panic disorder. A randomised, double-blind, placebo-controlled study. British Journal of Psychiatry, 167, 374-379.

\section{Pohl, R. B., Wolkow, R. M. \& Clary, C. M. (1998)} Sertraline in the treatment of panic disorder: a doubleblind multicenter trial. American Journal of Psychiatry, I55, 1189-1195.

Pollack, M. H., Otto, M.W., Worthington, J. J., et a (1998) Sertraline in the treatment of panic disorder: flexible-dose multicenter trial. Archives of General Psychiatry, 55, 1010-1016.

Riskind, J. H., Beck, A. T., Brown, G., et al (1987) Taking the measure of anxiety and depression: validity of the reconstructed Hamilton scales. Journal of Nervous and Mental Disease, 175, 474-479.
Schneier, F. R., Liebowitz, M. R., Davies, S. O., et al (1990) Fluoxetine in panic disorder. Journal of Clinical Psychopharmacology, 10, 119-121.

Shear, M. K., Brown, T. A., Barlow, D. H., et al (1997) Multicenter collaborative panic disorder severity scale. American Journal of Psychiatry, 154, 1571-1575.

Sheehan, D. V. (1983) The Anxiety Disease. New York: Charles Scribner and Sons.

Sheikh, J. I., Londborg, P., Clary, C. M., et al (2000) The efficacy of sertraline in panic disorder: combined results from two fixed-dose studies. International Clinical Psychopharmacology, 15, 335-342.

Spielberger, C. D. (1983) Manual for the State-Trait Anxiety Inventory. Palo Alto, CA: Consulting Psychologists.

Wade, A. G., Lepola, U., Koponen, H. J., et al (1997) The effect of citalopram in panic disorder. British journal of Psychiatry, I70, 549-553. 